\title{
A Randomized Double-blind Controlled Study on the Efficacy of Epidural Injection of Clonidine and Lidocaine and Their Combination in Patients with Chronic Pain
}

\author{
Dr. P. Gayatri ${ }^{1}$, Dr. B. Sowbhagya Lakshmi \\ ${ }^{1}$ Postgraduate in MD Anaesthesiology, RMC, Kakinada, India \\ ${ }^{2}$ Professor and HOD, Department of Anaesthesiology, RMC, Kakinada (Corresponding Author)
}

\begin{abstract}
Background: Pain is one of the common complications which causes delayed recovery and more extended hospital stay. Objective: To compare the safety and efficacy of postoperative analgesia with epidural Buprenorphine and Butorphanol. Methods: The study design was a prospective randomized controlled double-blinded clinical study.In total, 60 patients who belonged to the ASA physical status class I and II, aged between 30 - 50 years, scheduled for elective laparoscopic surgeries were randomly assigned into two groups; each group contains 30 patients. Surgeries were done under general anesthesia. At the end of the surgery, "Group A" Received $1 \mathrm{ml}(0.3 \mathrm{mg})$ of Buprenorphine diluted to $10 \mathrm{ml}$ with $\mathrm{NS}$ and "Group B" Received $1 \mathrm{ml}$ (1mg) of Butorphanol Tartrate diluted to 10ml with NS through the epidural catheter. Observations were recorded by a blind observer anesthesiologist. VAS was assessed every hour till the 6th hour, then 2nd hourly till the 12th hour. To asses sedation, the Ramsay sedation score (RSS) was used. Patients were given rescue analgesic (inj.Diclofenac $50 \mathrm{mg} I M$ ) when the patients complained of pain, and the pain scales (VAS) more than 6. The total duration of postoperative analgesia was noted. Patients were observed for any side effects like respiratory depression, nausea, vomiting, pruritus, and headache. Student's t-test and Mann-Whitney's tests were used for analysis. Results: There was a statistically highly significant difference in the duration of analgesia between the two groups (p<0.001). Conclusion: Epidural Buprenorphine significantly reduced pain \& increased the quality of analgesia with a longer duration of action compared to epidural Butorphanol tartrate.
\end{abstract}

Keywords: Epidural buprenorphine, epidural butorphanol tartrate, postoperative pain relief, laparoscopic surgeries

\section{Introduction}

Pain is becoming a major concern postoperatively as it gives rise to various psychological and physiological phenomena. Hence, effective pain control is crucial for too early mobilization and postoperative discharge.[1]The role of regional techniques, particularly epidural analgesia, is now well-established. When compared with conventional opioid analgesia, they provide earlier mobilization, superior analgesia, and earlier restoration of bowel function and reduced risk of postoperative thromboembolic and respiratory complications.Narcotic analgesic drugs are frequently used in epidural anesthesia.[2] Epidural administration of $\mu$-receptor opioid agonists like morphine is regarded by many as the "gold-standard" single-dose neuraxial opioid due to its prolonged duration of action and postoperative analgesic efficacy. However, it is associated with side effects such as pruritus, nausea, vomiting, urinary retention, and respiratory depression.[3,4]Buprenorphine is a semi-synthetic opioid with strong agonistic activity at the $\mu$ receptor and antagonistic activity at the $\kappa$ receptor.[5]Butorphanol is a lipid-soluble narcotic drug with strong $\kappa$-receptor agonist and weak $\mu$-receptor agonist/ antagonist activity. These have been frequently used for postoperative analgesia.[6]The analgesic efficacy of epidural butorphanol and buprenorphine is comparable to that of morphine, with less respiratory depression, pruritus, and nausea and vomiting. [7,8]

\section{Methods}

Following approval of the Ethical Committee of Rangaraya Medical College and Government General Hospital, Kakinada and obtaining written informed consent from 60 patients who belonged to the American Society of Anesthesiologists classes I-II, aged between 30 -50 years scheduled for elective laparoscopic surgeries were randomly assigned into two groups of 30 patients each.All patients provided written informed consent for the surgical procedure and anesthetic method.A single anesthesiologist handled all the anesthesia procedures.

All patients underwent pre-anesthetic evaluation on the previous day of surgery.Epidural catheters were secured for all the patients before giving general anesthesia.Surgeries were done under general anesthesia. At the end of surgery, in study Group A, $1 \mathrm{ml}(0.3 \mathrm{mg})$ of buprenorphine diluted to 10 $\mathrm{ml}$ with Normal saline was injected through the epidural catheter.

In Group B, $1 \mathrm{ml}(1 \mathrm{mg})$ of Butorphanol tartrate diluted to $10 \mathrm{ml}$ with Normal saline was injected through the epidural catheter. Patients were observed in the recovery room. Observations were recorded by a blinded observer anaesthesiologist. Visual analog pain scales (VAS) were assessed every hour till the 6th hour, then 2 nd hourly till the 12th hour. To assess sedation, the Ramsay sedation score (RSS) was used. The pulse rate, Blood pressure, and respiration were monitored. 
Patients were given rescue analgesic (inj. Diclofenac $50 \mathrm{mg}$ IM) when the patients complained of pain, and the pain scales (VAS) were more than 6.The total duration of postoperative analgesia was taken as the period from the time of administering epidural drugs until the patients first complained of pain, and the VAS was greater than 6 . Patients were observed for any side effects such as nausea, vomiting, pruritus, headache, and respiratory depression.

\section{Statistical Analysis}

Taking an alpha error 0.01 and a beta error of 0.01 for the parameter (duration of analgesia), the minimal sample size required to conduct the study would be 29/group. To compensate for greater variability, thirty (30) patients were included in each group.Data were analyzed, compiled, and presented as mean, standard deviation (SD), percentages, $t-$ test, and Mann-Whitney's test. The P-value was considered significant, as shown below:

P> 0.05- Not significant

$\mathrm{P}<0.05$ - Significant

$\mathrm{P}<0.001$ - Highly significant.

\section{Results}

Below Table showed that there was no difference in age and weight $(\mathrm{P}>0.05)$. Demographically, both the groups were compared using unpaired Student's t-test.

Table 1

\begin{tabular}{|c|c|c|c|}
\hline & Group A & Group B & Significance \\
\hline Number of patients & 30 & 30 & $\mathrm{p}$ \\
\hline Mean age+/-SD & $50.53+/-4.2$ & $51.67+/-4.5$ & 0.317 \\
\hline Mean weight+/-SD & $63.43+/-5.58$ & $64.07+/-3.8$ & 0.61 \\
\hline
\end{tabular}

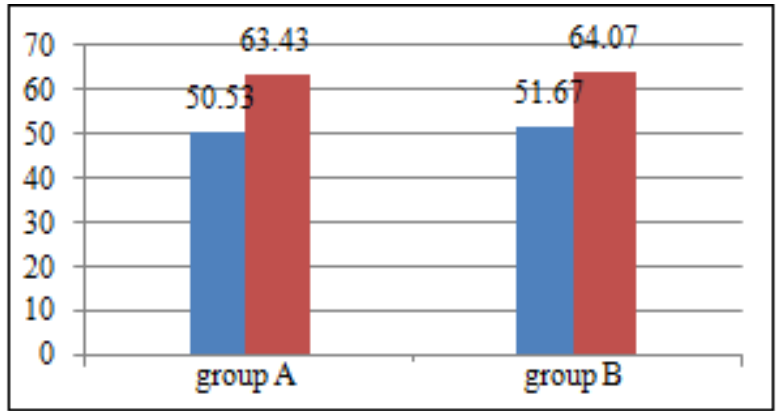

- The VAS among both the groups were compared using Mann-Whitney's test.

- Comparison of visual analog pain scores in both groups.

Table 2:

- The duration of analgesia was evaluated using Student's ttest.

- Duration of analgesia in minutes

Table 3

\begin{tabular}{|c|r|c|c|}
\hline & Group A & Group B & Significance (p) \\
\hline Mean +/- SD & $566.17+/-73.64$ & $325.53+/-47.42$ & $<0.001$ \\
\hline
\end{tabular}

- The RSS among the groups were compared using MannWhitney's test.
- Comparison of Ramsay sedation score (RSS) in both groups

Table 4

\begin{tabular}{|c|c|c|}
\hline Group & Mean rank & Significance(p) \\
\hline RSS 1 & & \multirow{2}{*}{$<0.001$} \\
\hline A & 29.6 & \\
\hline B & 43.77 & \multirow{2}{*}{$<0.001$} \\
\hline RSS 2 & & \multirow{2}{*}{$<0.001$} \\
\hline A & 31.52 & \\
\hline B & 42.25 & \\
\hline RSS 3 & & \multirow{2}{*}{} \\
\cline { 1 - 2 } A & 25.77 & \multirow{2}{*}{} \\
\hline B & 42.75 & \\
\hline
\end{tabular}

\section{Discussion}

Epidural opioids have been administered commonly to relieve anxiety and reduce pain associated with surgical procedure. Opioids bind to specified opioid receptors in the central \& peripheral nervous system. There are three (3) principle classes of opioid receptors like mu, delta, and kappa $(\mu, \delta$, and $\kappa)$ receptors. The pharmacodynamic response based upon which receptor the opioid binds, the affinity of binding, and whether it is an antagonist or an agonist. Buprenorphine is a semi-synthetic opioid drug with partial agonist activity at the $\mu$-receptor (mu), partial or full agonist activity at the $\delta$-receptor (delta), and competitive antagonist activity at the kappa receptor.It is a powerful analgesic, roughly 25-40 times as potent as morphine.[9]It is prepared in a preservative-free solution that has a strong affinity for opioid receptors and high lipid solubility. Butorphanol tartrate is a synthetic morphine derivative drug which has partial agonist and antagonist activity at the mu receptor and agonist activity at the kappa receptor.[9]As with other opioid analgesics, central nervous system effects (confusion, sedation, dizziness) are considerations with Butorphanol drug.Thus, this study was made to evaluate the efficacy of epidural Butorphanol tartrate and epidural buprenorphine for postoperative analgesia in laparoscopic surgeries.

Quality of analgesia (visual analog scale)In our study, as showed in table 2, Group A showed significantly higher mean rank of VAS at 1 st hr (39 vs. 22 [P < 0.001]) when compared to Group B, suggesting that onset of analgesia was earlier in Butorphanol group. From 2nd hr to 12th hr, we could observe a statistically significant decrease $(P<0.001)$ in the mean rank of VAS of the study Group A when compared to the study Group B showing that epidural buprenorphine significantly increased the quality of analgesia and reduced pain.

\section{Duration of analgesia}

In our study, Buprenorphine (Group A) had a longer duration of analgesia when compared to Butorphanol tartrate (Group B) for postoperative analgesia as in table 3, (566.17 \pm 73.64 vs. $325.53 \pm 47.42 \min [\mathrm{P}<0.001])$ 


\begin{tabular}{|c|c|c|}
\hline Group & Mean rank & Significance(p) \\
\hline VAP 1 & & \multirow{2}{*}{$<0.001$} \\
\hline A & 39 & \\
\hline B & 22 & \multirow{2}{*}{$<0.001$} \\
\hline VAP 2 & & \\
\hline A & 16.4 & \\
\hline B & 44.6 & \multirow{2}{*}{$<0.001$} \\
\hline VAP 3 & & \\
\hline A & 18.5 & \\
\hline B & 42.5 & \\
\hline
\end{tabular}

Side effects

Ramsey Sedation Score was (43.77-23.47) significantly higher with Butorphanol tartrate as compared to (29.6016.38) buprenorphine in our study.Another side effect of Respiratory depression was not seen in both the study groups. Nausea, vomiting, and headache were more in the buprenorphine group; however, pruritus was found to be more with Butorphanol. No other complication was observed in both groups.

\section{Conclusion}

Both Buprenorphine and Butorphanol tartrate can be used epidurally for effective and safe postoperative analgesia. However, epidural Buprenorphine significantly reduced pain and increased the quality of analgesia with a longer duration of action compared to epidural Butorphanol tartrate. Epidural buprenorphine had lesser sedation, but it caused more headache, nausea, vomiting. Pruritus was more with epidural Butorphanol. Respiratory depression had not occurred in both groups. No other complications were noted. Thus, we concluded that epidural Buprenorphine was a good alternative to epidural Butorphanol for providing postoperative analgesia.

\section{References}

[1] Revar B, Patel V, Patel B, Padavi S. A comparison of epidural butorphanol tartrate and tramadol hydrochloride for postoperative analgesia using csea technique. Int $\mathbf{J}$ Res Med. 2015;4:1-6.

[2] Kaur J, Bajwa SJ. Comparison of epidural butorphanol and fentanyl as adjuvants in the lower abdominal surgery: A randomized clinical study. Saudi J Anaesth. 2014;8:167-71.

[3] Sultan P, Gutierrez MC, Carvalho B. Neuraxial morphine and respiratory depression: Finding the right balance. Drugs. 2011;71:1807-19.

[4] Bromage PR, Camporesi EM, Durant, PA, Nielsen CH. Nonrespiratory side effects of epidural morphine. Anesth Analg. 1982;61:490-5.

[5] Jadon A, Parida S, Chakraborty S, Panda A. Epidural naloxone to prevent buprenorphine induced PONV. Internet J Anesth. 2007;16:1-4.

[6] Rosow CE. Butorphanol in perspective. Acute Care. 1988;12(Suppl 1):2-7.

[7] Wolff J, Carl P, Crawford ME. Epidural buprenorphine for postoperative analgesia.A controlled comparison with epidural morphine. Anesthesia. 1986;41:76-9.

[8] Parikh GP, Veena SR, Vora K, Parikh B, Joshi A. Comparison of epidural butorphanol versus epidural morphine in postoperative pain relief. Middle East $\mathbf{J}$ Anaesthesiol. 2014; 22:371-6.

[9] Gutstein HB, Akil H. Opioid analgesics. In: Brunton LL, Lazo JS, Parker KL, editors. The Pharmacological Basis of Therapeutics.Goodman and Gillman's. 11th ed. New York: Mcgraw Hill; 2006. pp. 279-378. Ch. 21. 